\title{
A comparative study of the effect of induction of labour with vaginal misoprostol versus prostaglandin E2 gel on the incidence of pathological cardiotocography tracing
}

\author{
Archana Kumari, Nikita Chauhan*, Anubha Vidyarthi
}

Department of Obstetrics and Gynecology, Rajendra Institute of Medical Sciences, Ranchi, Jharkhand, India

Received: 21 April 2020

Accepted: 27 April 2020

*Correspondence:

Dr. Nikita Chauhan,

E-mail: nikita.chauhan06@gmail.com

Copyright: () the author(s), publisher and licensee Medip Academy. This is an open-access article distributed under the terms of the Creative Commons Attribution Non-Commercial License, which permits unrestricted non-commercial use, distribution, and reproduction in any medium, provided the original work is properly cited.

\begin{abstract}
Background: The objective of the present study was to compare the two most commonly used agents for induction of labor-vaginal misoprostol and intracervical dinoprostone gel in terms of the incidence of cardiotocography (CTG) abnormalities and its correlation with fetal distress and fetomaternal outcome.

Methods: This is prospective case-control study conducted in department of obstetrics and gynecology, RIMS, Ranchi over a period of 15 months. 112 women requiring induction were randomly assigned to two groups of 56 each, Group M received vaginal misoprostol and Group D received intracervical dinoprostone E2 gel. 56 women with spontaneous labor served as control group. Groups were compared in terms of the incidence of suspicious or pathological CTG tracings, fetal distress, induction to vaginal delivery time, vaginal delivery rates, dose requirements, rate of emergency cesarean.

Results: Misoprostol was associated with shorter induction to delivery time ( 9.54 hours) than dinoprostone gel (13.54 hours), higher vaginal delivery rates $(80.35 \%$ versus $62.5 \%)$, higher delivery rates $(73.9 \%)$ with single dose itself unlike Group D, where $47.22 \%$ required more than one dose. Incidence of suspicious CTG was higher in group M $(15.68 \%$ ) versus $10.25 \%$ in Group D. Incidence of pathological CTG was also highest in Group M (7.8\%) followed by Group D $(2.56 \%)$ and Group C (7.8\%). Dinoprostone gel lead to failed induction in $25 \%$ women, and hence higher caesarean rates.

Conclusions: While misoprostol is a better agent for induction when compared with dinoprostone E2 gel in terms of induction-delivery time, higher vaginal delivery rates, less dose requirement, it is associated with greater incidence of non-reassuring/pathological CTG. There was justified improvement in perinatal outcome due to preparedness beforehand with use of CTG.
\end{abstract}

Keywords: Cardiotocography, Dinoprostone gel, Induction of labour, Prostaglandin E2, Vaginal misoprostol

\section{INTRODUCTION}

The goal of induction of labour is to achieve vaginal delivery by stimulating uterine contractions before the spontaneous onset of labour. Generally, induction of labour is a therapeutic option when the benefits of expeditious delivery outweigh the risks of continuing the pregnancy. ${ }^{1}$ The common indications include membrane rupture without labor, hypertensive disorders of pregnancy, oligohydramnios, post term pregnancy, and various maternal medical conditions such as chronic hypertension and diabetes. Oxytocin has been used for decades to induce or augment labour. Other effective methods include prostaglandins, such as misoprostol and dinoprostone, and mechanical methods that encompass stripping of membranes, artificial rupture of membranes, transcervical balloons, and hygroscopic cervical dilators. ${ }^{2}$ Labour itself is a stress factor for the fetus within the 
uterus. During active labour the integrity of the uteroplacental circulation and the frequency and intensity of uterine activity influence the acid-base status of the fetus. Electronic fetal heart rate monitoring (EFM) with CTG is commonly used means of fetal wellbeing assessment in labour and to identify hypoxia indirectly before brain damage has been incurred. The efficacy of EFM during labour is judged by its ability to decrease complications, such as neonatal seizures, cerebral palsy, or intrapartum fetal death, while minimizing the need for unnecessary obstetric interventions, such as operative vaginal or caesarean delivery. ${ }^{3-5}$

As induction of labour is on the rising trend, it is important to understand and devise method to assess the fetal wellbeing, diagnose fetal distress at earlier stage and corelate with feto-maternal outcome in cases undergoing induction of labour. At present, non- invasive CTG monitoring of FHR seems to be desirable and better than intermittent auscultation in this regard. With this background, the present study was conducted with the primary objective of comparing two most commonly used agents for induction of labor-vaginal misoprostol and intracervical dinoprostone gel in terms of the incidence of CTG abnormalities (suspicious or pathological CTG tracings) and its correlation with fetal distress. Secondary outcomes like time interval from induction to vaginal delivery, vaginal delivery rates within 12 and 24 hours, dose requirements, incidence of tachysystole ( $>5$ contractions in 10 minutes), rate of emergency caesarean section due to fetal distress or nonprogress were also compared in the two groups.

\section{METHODS}

This was a hospital based prospective case-control study conducted in department of obstetrics and gynecology, RIMS, Ranchi from $1^{\text {st }}$ August 2017 to $31^{\text {st }}$ October 2018 over a period of 15 months. Approval for the study was obtained from Institutional Ethics Committee, RIMS, Ranchi. 112 pregnant women requiring induction of labour and fulfilling the inclusion criteria were included in study.

\section{Inclusion criteria}

- Patient with an indication for induction of labor

- Willingness to participate in the study and those who gave consent

- $\quad$ Singleton fetus

- Cephalic presentation

- Gestational age of 37 weeks or more.

\section{Exclusion criteria}

- Previous cesarean section, or any previous surgery of the uterus

- Grand multipara

- Malpresentations (face, breech, transverse lie)

- Intrauterine death
- Fetal anomalies

- Evidence of fetal distress on pre-induction cardiotocographic monitoring

- Any other contraindications for induction of labor (cephalo-pelvic disproportion).

Study group were randomly assigned into 2 groups of 56 women each. Group D received $0.5 \mathrm{mg}$ of prostaglandin E2 gel given intra-cervically every 6 hours to maximum 3 doses. Group $\mathrm{M}$ received 25 microgram of vaginal misoprostol 4 hourly to maximum of 4 doses. Fifty-six women who underwent spontaneous labour served as control (Group C.) All three groups were similar with respect to age, parity and gestational age. Informed consent was taken from all women. Pre-induction cardiotocography was performed and Bishop score was assigned on admission for all women enrolled in the study before randomization. Women with an established uterine contraction pattern of greater than 3 contractions in 10 minutes were not redosed with study agent. CTG monitoring was done 1 hour after induction, four hourly and intrapartum continuous monitoring once the patient was in active phase of labour. Vaginal examinations were performed 4 hourly. Uterine hyperstimulation were managed with a single dose of subcutaneous terbutaline (0.25) along with position change and oxygen administration. Cardiotocographic tracings were independently reviewed and graded into Normal, Suspicious or Pathological according to NICE guideline. ${ }^{6}$

Redosing was not done in case of suspicious or pathological CTGs. The decision to discontinue labour and deliver by LSCS was made if fetal distress supervened or delayed progress of labor occurred. At delivery, condition of the babies was assessed in terms of Apgar score at 1 and 5 minutes, birth weight and immediate neonatal nursing care provided. Perinatal morbidity and NICU stay and its outcome were monitored for fetal outcome. Maternal morbidity and hospital stay were recorded to identify the effect of induction on mother.

\section{Statistical analysis}

Descriptive statistical analysis has been carried out in this study. Results on continuous measurements are presented on Mean \pm SD and results on categorical measurements in number (\%). A p-value of less than 0.05 was considered significant for the purpose of result analysis. Student Ttest, chi-square test and fisher exact test have been used to calculate the p-value and data was analysed using SPSS.

\section{RESULTS}

Table 1 shows that most common indication for induction of labour in two study Groups $\mathrm{M}$ and $\mathrm{D}$ was prelabour rupture of membranes followed by post-dated pregnancy and HDP and were statistically similar. 
Table 1: Indications for induction.

\begin{tabular}{|c|c|c|c|c|}
\hline \multirow{2}{*}{ Indications } & \multicolumn{2}{|l|}{ Study group } & \multirow{2}{*}{ Total $(n=112)$} & \multirow{2}{*}{ p-value } \\
\hline & Group M $(n=56)$ & Group D (n=56) & & \\
\hline Hypertensive disorders of pregnancy & 2 & 5 & 7 & \multirow{4}{*}{0.595} \\
\hline Post-dated pregnancy & 24 & 23 & 47 & \\
\hline Pre-labor rupture of membranes & 29 & 28 & 57 & \\
\hline Rh negative & 1 & 0 & 1 & \\
\hline
\end{tabular}

Table 2: Analysis of Bishop scores.

\begin{tabular}{|llllllll|}
\hline \multirow{2}{*}{ Bishop score at } & Group $\mathbf{M}(\mathbf{n}=\mathbf{5 6})$ & & \multicolumn{2}{l}{ Group $\mathbf{D}(\mathbf{n}=\mathbf{5 6})$} \\
& Number of patients & Mean & SD & Number of patients & Mean & SD & p-value \\
\hline Baseline & 56 & 4.20 & 0.67 & 56 & 3.77 & 1.03 & 0.010 \\
\hline After $1^{\text {st }}$ dose & 28 & 7.14 & 2.49 & 41 & 5.15 & 1.90 & 0.001 \\
\hline After $2^{\text {nd }}$ dose & 6 & 8.83 & 2.32 & 26 & 5.73 & 2.25 & 0.020 \\
\hline
\end{tabular}

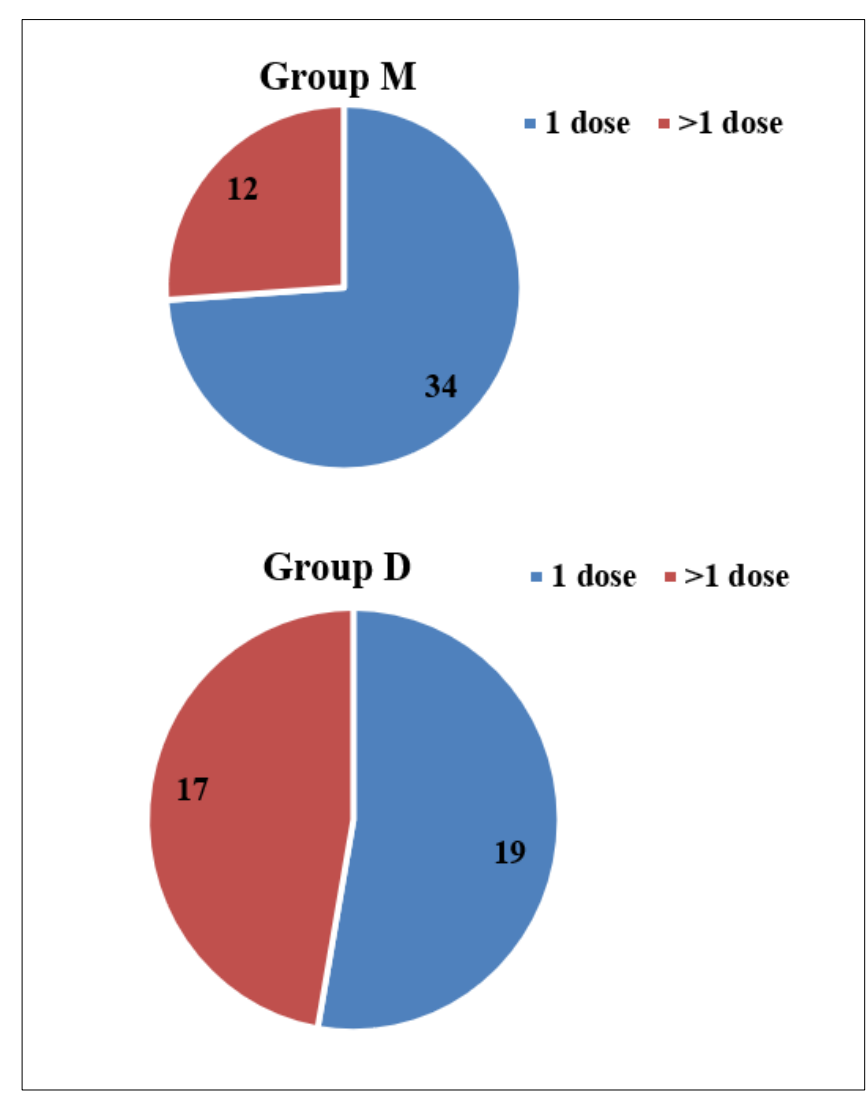

Figure 1: Dose required.

Table 2 shows that favourable mean bishop scores were achieved faster in Group $\mathrm{M}$ as compared with Group D with each dose.

Figure 1 depicts that 34 women (73.9\%) in group $\mathrm{M}$ and 19 women $(52.77 \%)$ in Group D delivered vaginally with single dose itself. Therefore, more successful vaginal deliveries with single dose was seen in Group M. Chi square test applied, and p-value was found to be statistically significant $(\mathrm{p}=0.0469)$. Figure 2 shows that out of 56 women, 51 women in Group M and 34 women in Group D went into early labour.

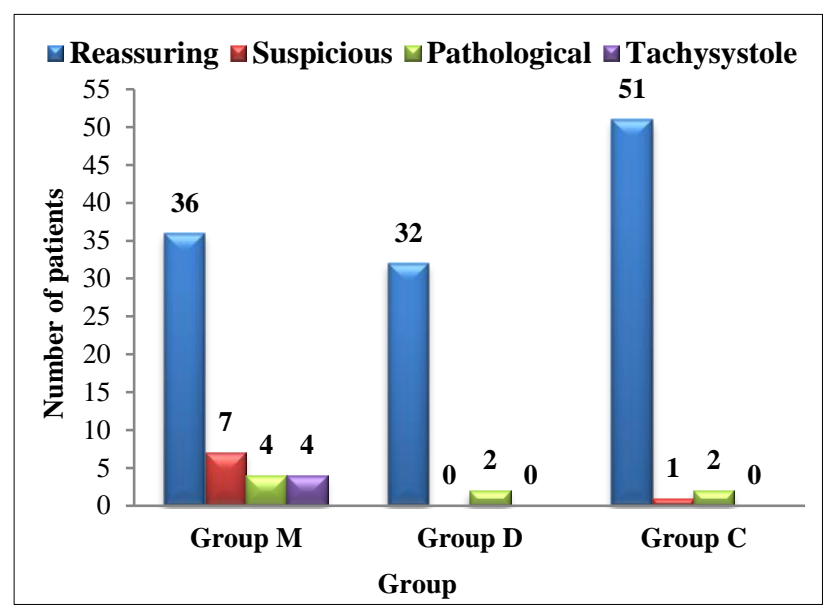

Figure 2: CTG in early labor.

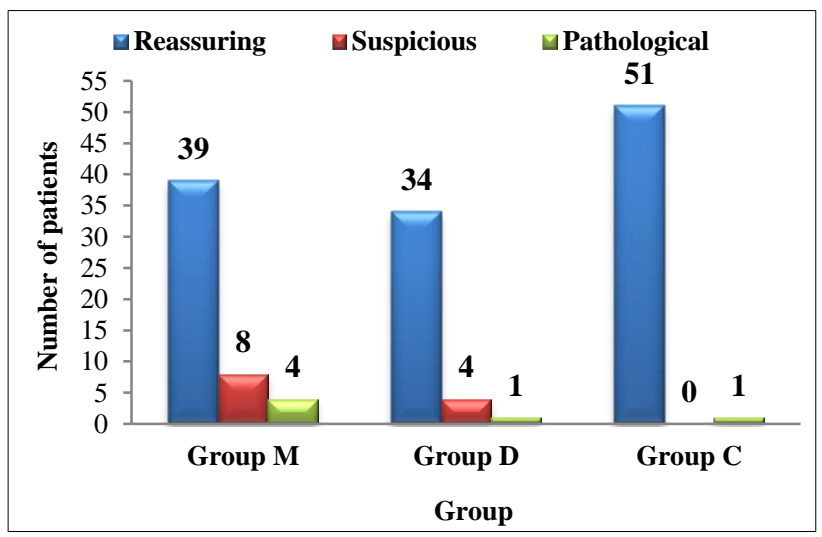

Figure 3: CTG in active labour.

Incidence of suspicious CTG was higher in Group $\mathrm{M}$ (13.7\%) as compared to Group D (0). Pathological CTG in group $\mathrm{M}$ was also higher $7.8 \%$ versus $2.58 \%$ in Group 
D. Tachysystole was seen in four cases in Group $M$ (7.8\%). No cases of tachysystole were observed in Group D. In Group C, two (3.57\%) pathological and one (1.7\%) suspicious CTG was seen. These values were found to be statistically significant (p-value 0.005). Figure 3 shows that CTG in 51 cases of Group $\mathrm{M}$ and for 34 cases in Group D who went into active labour. Incidence of suspicious CTG was highest in Group M (15.68\%) followed by Group D (10.25\%).

Incidence of pathological CTG was also highest in Group M (7.8\%) followed by Group D $(2.56 \%)$ and Group C (7.8\%). These values were found to be statistically significant (p-value 0.021).

Table 3: Mode of delivery.

\begin{tabular}{|c|c|c|c|c|}
\hline & Group M (n=56) & Group D $(n=56)$ & Group C $(n=56)$ & p-value \\
\hline Vaginal delivery & $45(80.35 \%)$ & $35(62.5 \%)$ & $48(85.71 \%)$ & \multirow{4}{*}{0.0460} \\
\hline Instrumental delivery & $1(1.78 \%)$ & $1(1.78 \%)$ & $1(1.78 \%)$ & \\
\hline LSCS & $10(17.85 \%)$ & $20(35.71 \%)$ & $7(12.5 \%)$ & \\
\hline Total & 56 & 56 & 56 & \\
\hline
\end{tabular}

Table 4: Induction-delivery interval.

\begin{tabular}{|llll|l|}
\hline \multirow{2}{*}{ Group } & Number of women & \multicolumn{2}{l|}{ Induction to delivery interval } & p-value \\
\cline { 1 - 4 } & & Mean (hours) & SD & 0.003 \\
\hline Group M & 46 & 9.54 & 4.13 & 0.89 \\
\hline
\end{tabular}

Table 5: Indications for caesarean section in three groups.

\begin{tabular}{|llll|}
\hline Indication & Group M (n=56) & Group D (n=56) & Group C (n=56) \\
\hline Failed induction & $1(10 \%)$ & $13(65 \%)$ & 0 \\
\hline Suspicious CTG & 0 & $3(15 \%)$ & 0 \\
\hline Pathological CTG & $7(70 \%)$ & $4(20 \%)$ & $3(42.8 \%)$ \\
\hline Arrest in stage II of labour & $2(20 \%)$ & 0 & $2(28.57 \%)$ \\
\hline NPOL & 0 & 0 & $3(42.85 \%)$ \\
\hline Total & $10(\%)$ & $20(\%)$ & $7(\%)$ \\
\hline
\end{tabular}

Table 6: Meconium stained liquor in Group $M$ and Group D.

\begin{tabular}{|llllll|}
\hline Meconium & Group M $(\mathbf{n}=\mathbf{5 6})$ & Group D $(\mathbf{n}=\mathbf{5 6})$ & Group C $(\mathbf{n}=\mathbf{5 6})$ & Total & p-value \\
\hline Yes & $20(35.71 \%)$ & $8(14.28 \%)$ & $10(17.85 \%)$ & 38 & 0.014 \\
\hline No & $36(64.28 \%)$ & $48(85.71 \%)$ & $46(82.14 \%)$ & 130 & \\
\hline Total & 56 & 56 & 56 & 168 & \\
\hline
\end{tabular}

Table 3 shows mode of delivery in different groups. In Group M, $80.35 \%$ of cases delivered vaginally unlike Group D where only $62.5 \%$ cases had a vaginal delivery. In Group C, $83.9 \%$ of the women delivered vaginally. Table 4 depicts that in cases of successful vaginal deliveries in Group M (46) and Group D (35), mean induction to delivery interval was found to be shorter in Group M (9.54 hours) than Group D (13.45 hours), which was statistically significant (p-value 0.003). Table 5 shows that in Group M, most common indication for LSCS was pathological CTG (70\%) followed by arrest in $2^{\text {nd }}$ stage of labour $(20 \%)$ and failed induction $(10 \%)$, unlike in Group D, where the most common indication was failed induction (65\%) followed by pathological CTG $(20 \%)$ and suspicious CTG (15\%).

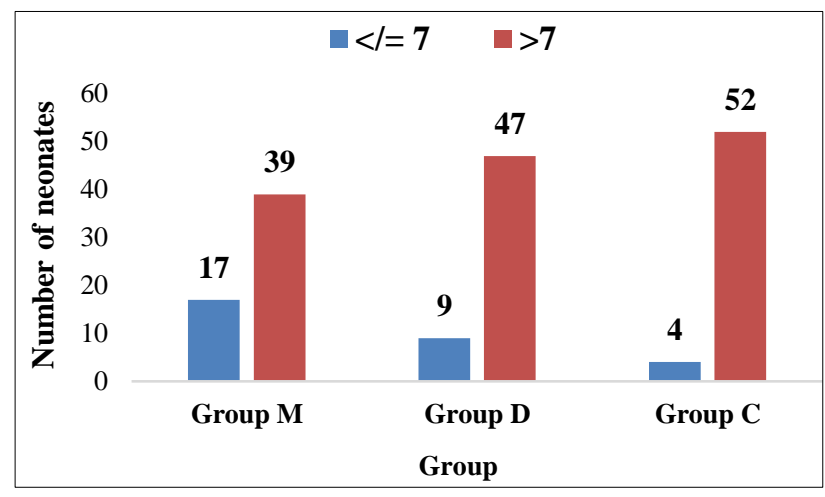

Figure 4: Apgar Scores at 1 minute in the three groups. 
In Group C, non-progress of labour (42.8\%) and pathological CTG $(42.8 \%)$ were common indications followed by arrest in $2^{\text {nd }}$ stage of labour $(28.57 \%)$. Overall, incidence of failed induction was much higher in Group D 13 out of $56(23.21 \%)$ when compared with Group M 1 out of 56 cases (1.78\%).

Figure 4 and 5 shows that Apgar scores were less than seven in $30.35 \%$ cases in Group $M, 16.07 \%$ cases in group D and $7.14 \%$ cases in Group C. This was found to be statistically significant (p-value 0.0053 ). However, at 5 minutes, Apgar scores of the three groups were statistically similar.

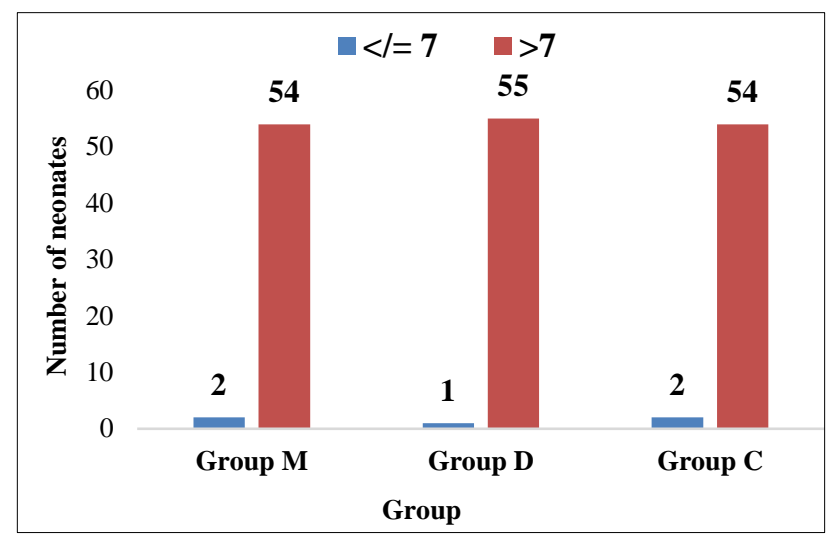

Figure 5: Apgar Scores at 5 minutes in the three groups.

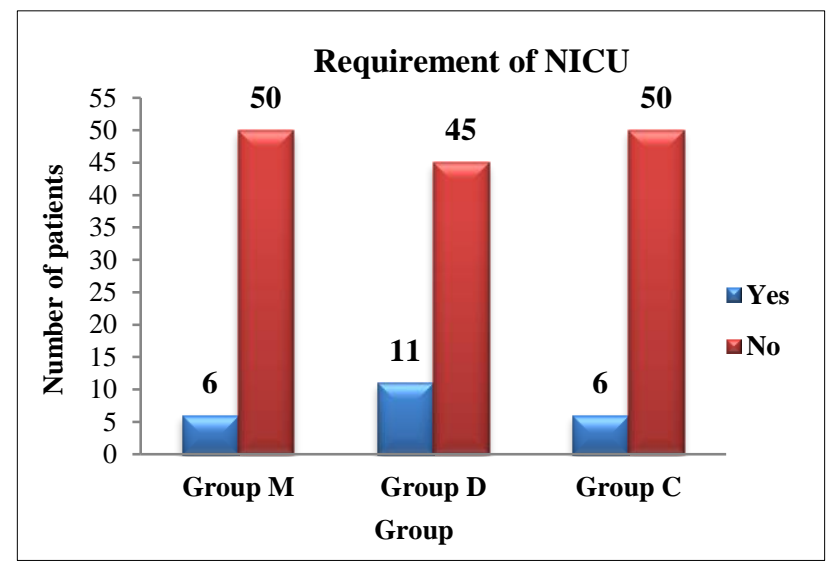

Figure 6: NICU admission in three groups.

Table 6 depicts that incidence of meconium stained liquor was highest in Group M $(35.71 \%)$ followed by Group C $(17.85 \%)$ and Group D (14.28\%). This difference was found to be statistically significant (p-value 0.014).

In Group M, incidence of neonates requiring resuscitation was higher (19.64\%) compared to other 2 Groups D and $\mathrm{C}$, where incidence was $8.9 \%$. This difference was not found to be statistically significant. Among the three groups, Group $\mathbf{M}$ had the highest incidence of babies requiring NICU admission at birth (19.64\%). Group D and Group $\mathrm{C}$ had equal number of NICU admissions
(10.71\%). However, this difference was not found to be statistically significant (Figure 6).

\section{DISCUSSION}

Induction of labour remains a challenge when it comes to unfavorable cervix. Prostaglandins are highly efficacious agents for this purpose, resulting in effective cervical ripening. Use of prostaglandins shortens inductiondelivery time and improves the probability of a successful vaginal delivery. In this study, authors compared the effect of vaginal misoprostol and intracervical prostaglandin E2 gel for induction of labour on CTG tracings. This study also compared these findings with incidence of non-reassuring CTG in women with spontaneous onset of labour. Use of these agents is associated with a higher incidence of abnormal uterine contractions and non-reassuring fetal heart rate patterns. ${ }^{7,8}$

Most common indication for induction in this study in both Group $\mathrm{M}$ and Group D was pre-labour rupture of membranes $(51.7 \%$ and $50 \%$, respectively) followed by post-dated pregnancy $(42.8 \%$ and $41 \%$, respectively). However, PROM was not a common indication in previous studies. In a study conducted by Rayburn Miller et al major indication was post-dated pregnancy (22.5\%) in misoprostol group and PIH (15\%) in dinoprostone group. ${ }^{9}$ Perry and Leaphart et al reported post-dated pregnancy as most common indication (misoprostol group $17.5 \%$ and dinoprostone group 14\%), similar to Stempel et al study (25\% and $22.7 \%$ in dinoprostone and misoprostol groups, respectively). ${ }^{10,11}$ In a recent study conducted by Goetzl L, PROM and post-dated pregnancy were common indications for induction of labour. ${ }^{12}$ Average Bishop score in Group M was 4.20 \pm 0.67 , and in Group D, it was 3.77 \pm 1.03 . Similar Bishop scores on admission were seen in study by Perry and Leaphart et al (Group M 2.5 and Group D), and Stempel et al (Group M $3.34 \pm 1.73$, Group D 3.69 \pm 1.43$).{ }^{10,11}$ A faster and favorable change in mean Bishop score was seen in undelivered cases in Group M. In this study, it was observed that this faster change in Bishop score in Group $\mathrm{M}$ favored vaginal delivery unlike Group D, where mean change in Bishop score was not significant with each dose. Similar observation was made by Agarwal $\mathrm{N}$ et al, where they also observed better rise of Bishop score in Group M. ${ }^{13}$

In Group M, $73.9 \%$ of cases delivered vaginally with single dose itself unlike in Group D, where $47.22 \%$ of the cases required more than one dose similar to the study by Rowland $\mathrm{S}$ et al. ${ }^{14}$ It was observed that induction to delivery time was significantly shorter in Group M (9.53 hours) as compared to Group D (13.50 hours). Ozkan S et

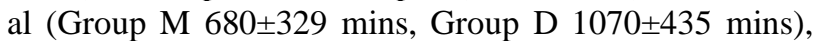
Chitrakar NS (Group M 3.91 hours), Rowland S et al (Group M 925.8 minutes, Group D 1577.6 minutes) also reported shorter induction-delivery time with misoprostol. ${ }^{14-16}$ 
In this study, it was observed that cardiotocographic abnormalities were more frequent when misoprostol was used as induction agent instead of prostaglandin E2 gel. Incidence of non-reassuring CTGs was higher in women undergoing induction of labour as compared to women in spontaneous labour. Higher incidence of Tachysytole was also seen in Group M.

Incidence of non-reassuring or abnormal features like early decelerations (Group M: 17.86\%, Group C: 12.5\%, Group D: $10.7 \%$ ), late decelerations (Group M: 30\%, Group D: $12.5 \%$, Group C $3.5 \%$ ), decreased beat to beat variability (Group M: 7.14\%, Group D: 3.57\%, Group C: $3.57 \%$ ) were significantly higher in Group M. Variable decelerations with concerning characteristics were observed in 2 women each belonging to Group D and Group M. Tachycardia and prolonged deceleration were seen in Group $\mathrm{M}$ only (incidence of each being 1.7\%). Apart from the CTG abnormalities, incidence of tachysystole was very high in Group M (7.14\%), whereas, it was only $1.7 \%$ in Group D. Studies conducted by Ramsey et al (hyperstimulation $2.9 \%$ in Group D versus $13.2 \%$ in Group M), Wing et al (Tachysystole: Group M 36.8\% Group D 11.9\%; hyperstimulation Group M 5.8\% and Group D 3\%), Sanchez-Ramos et al (Group M 34.4\%, Group D 13.8\%) also reported higher incidence of tachysystole in Misoprostol group. ${ }^{7,17,18}$

Though frequent cardiotocographic abnormalities were seen in Group M, the cesarean rate was higher in Group D. This could be because though cardiotocographic abnormalities were seen with the misoprostol group, they had a very favorable Bishop score with rapid progression of labour unlike Group D where incidence of failed induction was very high. Thirteen $(23.21 \%)$ women being induced with prostaglandin E2 gel underwent caesarean section for failed induction which was much higher as compared to Group M where only one (1.7\%) case of failed induction was observed. Rate of cesarean section was found to be highest in group D $(35.71 \%)$ followed by Group M (17.85) and Group S (12.5\%).

In Group M, most common indication for LSCS in this study was pathological CTG $(70 \%)$ followed by arrest in $2^{\text {nd }}$ stage of labour $(20 \%)$ and failed induction $(10 \%)$, unlike Group D, where the most common indication was failed induction (65\%) followed by pathological CTG (20\%) and suspicious CTG (15\%). In Group C, nonprogress of labour $(42.8 \%)$ and PCTG (42.8\%) were common indications followed by arrest in $2^{\text {nd }}$ stage of labour $(28.57 \%)$. Similar observation of higher caesarean delivery rate was made in studies conducted by Stempel et al (Group D 18\%, Group M 15.9\%) and Perry et al (Group D 14.3\% and Group M 11.1\%). ${ }^{10,11}$

The study shows $30.5 \%$ of neonates in Group M, $16.07 \%$ of neonates in Group D and 7.14\% of neonates in Group $\mathrm{C}$ had an Apgar score of $\leq 7$. These values were found to be statistically significant. However, with immediate resuscitative measures, Apgar scores at 5 minutes were found to be comparable with only $3.57 \%$ neonates in Group M, $1.7 \%$ neonates in Group D and $3.57 \%$ neonates in Group $\mathrm{C}$ with Apgar scores $\leq 7$. Many studies have reported that there was no statistically significant difference in the Apgar scores of neonates in Group M and Group D. ${ }^{9,17,36}$ The incidence of meconium stained liquor was highest in Group M $(35.71 \%)$ followed by Group D (14.28\%) and Group C (12.5\%). Whether this difference was due to increased fetal distress in the misoprostol group or due to a direct effect of misoprostol on the fetal gastrointestinal tract is unknown. Wing et al observed that there was a higher prevalence of meconium passage in the misoprostol group $(27.9 \%)$ than in the dinoprostone group $(10.5 \%)(\mathrm{p}<0.05)$, unlike Agarwal $\mathrm{N}$ et al, who did not observe a significant difference in meconium stained liquor in Groups $\mathrm{M}$ and Group $\mathrm{D}$ in their study. ${ }^{13,17}$

The study shows vaginal misoprostol was associated with higher incidence of NICU admission (7.14\%) compared to dinoprostone gel $(5.35 \%)$ and those with spontaneous onset of labor $(3.57 \%)$. However, these observations were not found to be statistically significant. Similar findings were observed in other studies as well where Group M and Group D were found to be similar with respect to neonatal outcomes. Meyer et al observed that there was no statistically significant difference in neonatal outcome. ${ }^{19}$

\section{CONCLUSION}

It is important to understand the effect of two commonly used agents for induction of labor on feto-maternal outcome and also to devise methods to prevent any untoward effect of induction of labor with these two agents.

With this study, it can be concluded that while vaginal misoprostol is a very good agent for induction of labor as it is associated with shorter induction-delivery time, higher vaginal delivery rates and successful vaginal deliveries with single dose and shorter hospital stay. Prostaglandin E2 gel was associated with a significantly higher rate of cesarean sections mainly due to failed induction which also leads to prolonged hospital stay and higher maternal morbidity. In a developing country like India, vaginal misoprostol offers an advantage in terms of being cheaper than prostaglandin E2 gel. Cold chain is not required for storage of misoprostol, can be kept at room temperature unlike prostaglandin E2 gel which is again advantageous in poor resource setting.

However, unlike prostaglandin E2 gel, misoprostol is associated with greater incidence of suspicious and pathological CTG tracing and tachysystole. These nonreassuring CTG findings were found to be associated with meconium stained liquor, Apgar scores $<7$ at 1 minute, requirement of resuscitation and NICU admission which are all parameters that indicate intrauterine hypoxia of the fetus and fetal distress. However, with 
timely resuscitative measures, Apgar scores at 5 minutes returns to normal. Thus, this study also indicated that CTG abnormalities have an association with the outcome of the fetus in women undergoing induction of labor. Continuous electronic fetal monitoring during induction of labor helps to understand fetal status better and predict fetal distress and fetal outcome more accurately as compared with auscultation alone. Preparedness beforehand for proper and timely resuscitation can help the neonate recover from transient effect of inducing agents and labor itself.

\section{Funding: No funding sources}

Conflict of interest: None declared

Ethical approval: The study was approved by the Institutional Ethics Committee

\section{REFERENCES}

1. American College of Obstetricians and Gynecologists: Induction of labor. Practice Bulletin No. 107, 2009, Reaffied; 2013.

2. Cunningham F, Gary. Kenneth J, Leveno. Steven L. Bloom. Spong Yet al; Induction and Augmentation of Labor; Williams Obstetrics, $24^{\text {th }}$ edition; Washington DC, McGraw-Hill; 2014:523-534.

3. Rayburn WF. Pre-induction cervical ripening: basis and methods of current practice. Obstet Gynecol Surv. 2002;57(10):683-92.

4. Adamsons K, Myers Re. Late decelerations and brain tolerance of the fetal monkey to intrapartum asphyxia. Am J Obstet Gynecol. 1997;128:893.

5. American College of Obstetricians and Gynecologists. Intrapartum fetal heart rate monitoring. ACOG Pract Bulletin. 2005;105(5):1161-7.

6. NICE. Interpretation of cardiotocograph traces. Intrapartum Care: NICE Guideline CG; 2014:190.

7. Sanchez-Ramos L, Kaunitz AM, Wears RL, Delke I, Gaudier FL. Misoprostol for cervical ripening and labor induction: a meta-analysis. Obstet Gynecol. 1997;89:633-42.

8. American College of Obstetrician and Gynecologists. Response to Searle's drug warning on misoprostol. Committee opinion 248. Washington, DC: ACOG; 2000.

9. Miller AM, Rayburn WF, Smith CV. Patterns of uterine activity after intravaginal PGE2 during ppreinduction cervical ripening. Am J Obstet Gynaecol. 1991;165:1006-9.
10. Perry MY, Leaphart WL. Intracervical versus posterior fornix dinoprostone insert for induction of labor. Am J Obstet Gynaecol. 2004;103:13-7.

11. Stempel JE, Prins RP, Dean S. Pre-induction cervical ripening: a randomized prospective comparison of the efficacy and safety of intravaginal and intracervical PGE2 gel. Am J Obstet Gynaecol. 1997; 176:1305-12.

12. Goetzl L. Methods of cervical ripening and labor induction: Pharmacologic. Clinical Obstetrics and Gynecol. 2014;57:377-90.

13. Agarwal N, Gupta A, Kriplani A, Bhatla N, Parul. Six hourly vaginal misoprostol versus intracervical dinoprostone for cervical ripening and labor induction. J Obstet Gynaecol Res. 2003;29:147-51.

14. Rowlands S, Bell R, Donath S, Morrow S, Trudinger BJ. Misoprostol versus dinoprostone for cervical priming prior to induction of labour in term pregnancy: a randomised controlled trial. Aust $\mathrm{N} \mathrm{Z} \mathrm{J}$ Obstet Gynaecol. 2001;41(2):145-52.

15. Ozkan S, Çalışkan E, Doğer E, Yücesoy İ, Özeren S, Vural B. Comparative efficacy and safety of vaginal misoprostol versus dinoprostone vaginal insert in labor induction at term: a randomized trial. Arch Gynecol Obstet. 2009;280(1):19-24.

16. Chitrakar NS. Comparison of Misoprostol versus Dinoprostone for pre-induction cervical ripening at term. J Nepal Health Res Counc. 2012;10(1):10-5.

17. Wing DA. A comparison of misoprostol and prostaglandin E2 gel for preinduction cervical ripening and labor induction. Am J Obstet Gynecol 1995 Jun;172(6):1811-6.

18. Ramsey PS, Jones MM, Rahall A, Goodwin TM, Paul RH. Cardiotocographic abnormalities associated with dinoprostone and misoprostol cervical ripening. Obstet Gynecol. 2005;105(1):85-90.

19. Meyer M, Pflum J, Howard D. Outpatient misoprostol compared with dinoprostone gel for preinduction cervical ripening: A randomized controlled trial. Obstet Gynecol. 2005;105:466-72.

Cite this article as: Kumari A, Chauhan N, Vidyarthi A. A comparative study of the effect of induction of labour with vaginal misoprostol versus prostaglandin E2 gel on the incidence of pathological cardiotocography tracing. Int J Reprod Contracept Obstet Gynecol 2020;9:2274-80. 